\title{
A Left-Hand-Focused Analysis of Piano Beginning Methods
}

\author{
İrem Çelikten Hepgüler \\ State Conservatory, Anadolu University, PO Box 26050, Eskişehir, Turkey
}

\begin{abstract}
One of the most critical elements in musical instrument education is the selection of the appropriate method. Many parameters are taken into consideration in the selection of the method (the student's age, his/her physical features, ability, etc.). The accuracy of the method depends on the teaching method of the instructor. It is simply impossible to have only one accurate method that is applicable for everyone and every context. The purpose of the methods is to develop technical capabilities and also to acquire theoretical knowledge. Exercises play a key role in piano education and literature. In this study, to compare different left-hand ideas in piano beginning methods, the beginner-level methods of five different countries were examined with a left-hand focus.
\end{abstract}

Keywords: piano, beginning method, left hand

DOI: $10.7176 / \mathrm{ADS} / 96-01$

Publication date: November $30^{\text {th }} 2021$

\section{Introduction}

One of the most important factors supporting the correct technique in music instrument training is the method, following the correct approach of the trainer. Successful learning is achieved only when these two parameters are correctly combined. Each method has its own exercises and methods, whose various uses are at the discretion of the instructor. Diversity in practice and study methods, rather than a uniform practice system, produces better success rates.

In this study, first of all, the periods up to the method selection in the beginner piano education were examined because, without the basic knowledge of the instrument, the student's education may progress incompletely and incorrectly. Deep and detailed study forms the basis of instrument training. Without correct sitting, posture, and hold, instrument training cannot be expected to go smoothly. In this study, the selected methods were also examined by taking these factors into account. A method cannot be expected to teach what needs to be taught physically because this is the instructor's task. However, it is important for young students to work with a visual method related to hand position and sitting, both in terms of encouragement and retention.

After the person who is to learn to play the piano has acquired the basic skills, the most important step is the choice of method. This selection phase varies from person to person. However, each person is taught some skills, such as hand posture and sitting properly, in the same way. In the current study, beginner-level piano methods were examined with a left-hand focus. This is because learning to play it with the right hand usually starts with the first finger. However, this may vary considerably regarding the left hand. Thus, the methods used by various nations were examined by focusing on the left hand, and the methodological differences between them were identified.

\section{The Beginning of Piano Education}

The first thing to do when starting piano education is to introduce the instrument to the student. This stage is very encouraging for both amateur and professional training. In addition, if a person is going to learn to play an instrument, knowing all its features should be natural.

After introducing the piano to the student, next step is teaching the sitting position. This is the first step of a habit that the learner will continue for the rest of his/her life. It is of essence that the correct posture is learned well because there will be no chance to go back to correct it later. An incorrectly learned sitting position may cause not only a bad appearance but also some health problems. Therefore, it is an issue that needs serious attention. Incorrect sitting also directly affects playing an instrument. Students who cannot use and move their body comfortably experience difficulties in the education process, which may even affect interpretation of a musical composition.

After these most basic steps, comes the stage of choosing or creating a method. At this stage, although the amateur vs. professional difference is not prominent at first, a big difference will emerge in a short time. In this study, the first stage, in which there is not much difference in general, will be discussed. The skills that the student should learn as a technical priority will be overviewed and discussed.

\subsection{Method Selection Stage}

The most important idea at the beginning of instrument education is to teach the technique correctly. The wrong technique taken at the beginning becomes a very difficult or unsolvable problem later on. The only way to achieve this accuracy is to follow the right methods and exercises. There are many known and used methods. 
The accuracy and usefulness of the content of these methods as a whole is debatable. In the method selection stage, some criteria should be determined first. Whether a person will receive an amateur or professional education is also a very important factor in determining these criteria. It is wrong to start and continue education based on only a certain method. In particular, the idea of fully covering a textbook and teaching all of its content, as many instructors do, limits instructional variety. This should be done only when one is absolutely sure of the diversity and technical functionality of the method. The necessity of benefiting from many sources, especially at the initial stage, will positively affect the process of music education.

\subsection{Learning the Hand Position Correctly}

After learning the sitting position, the second step is to learn the hand position correctly. This stage is directly proportional to the physique of the student. A person whose hand is not suitable for playing the piano can only continue his/her musical interest as an amateur. With the proliferation of instrument-playing courses in the $2000 \mathrm{~s}$, the instrument lessons taught by non-professionals have resulted in incorrect hand positions that are difficult to correct. In piano training, it is very important to learn the hand position correctly in a timely manner.

In many piano lessons, including those applying the well-known methods, the left hand starts playing with the fifth finger or the first finger. Starting with the fifth finger is an incorrect position, because of the physical weakness of this finger. A student who has just started piano education cannot be expected to press the weakest finger strongly without changing the correct hand position. For this reason, it is incorrect to start with the fifth finger of the left hand in two-hand studies, even if separate hand exercises were done before. Instead, it will be more efficient to start with the third finger when the left hand is in a natural position. This proposal eliminates the first studies of many known methods at the method selection or creation stage. Rather than assuming that the whole book must be studied, being goal-oriented yields better results.

After the exercises studied with separate hands in the first studies in piano training (especially at this stage, the fifth finger should not be the focus, the first basic exercise can be a study in which both hands play the same note and aim to achieve wrist control. This will be very helpful for early learning of hand and wrist control. During this practice, the child can be shown the 'cat walk,' which is done by moving the hands and wrists freely in front of each other. Besides being fun for the child, this exercise aims to teach the wrist movement naturally. While this exercise can be done with all fingers, it is most effective with the third fingers in terms of the natural position of the hand. This is the simplest exercise that can be done on any note or octave before the child learns the notes yet. At the same time, if desired, training can be initiated with this simple exercise without working with separate hands. The student internalizes the wrist movement learned by imitation at the beginning of the training and uses it in such positions. This study also aims to eliminate the problem of curling the first knuckle of the finger, which is a major problem in piano playing. While the student slowly experiences this imitation movement, s/he can easily control her/his fingers and make this behavior permanent.

\subsection{Learning the Clefs}

One of the most important problems when starting piano training is that both hands play different clefs, which is quite confusing for many people. In most methods, both hands start playing with G-clef, which actually aims to facilitate learning, but it prolongs the learning process. The student who gets used to reading the G-clef in the left hand will have even more difficulty in learning the F-clef. Instead, the student must learn from the beginning to play different clefs in two hands. Surely, the student can practice different hands, provided that they are in different clefs beforehand. Methods in which two hands are started to play in the G-clef are not included in this study.

\section{Analysis of Methods}

3.1 Oscar Beringer (A Complete Pianoforte Tutor, the UK)

In this method, the first exercises are written for separate hands. Both hands do the same exercise in opposite directions with the same finger number on different clefs. Both hands start with the first finger, but unlike many other methods, it starts with the $\mathrm{G}$ note instead of the middle $\mathrm{C}$. The first finger to play holds the note played, forming a support for the other finger to play. This exercise is a good exercise for finger strengthening, especially for weak fingers. However, it may be too early for those who have just started playing the piano, considering that it can strain the weak fingers of the left hand. 
I.
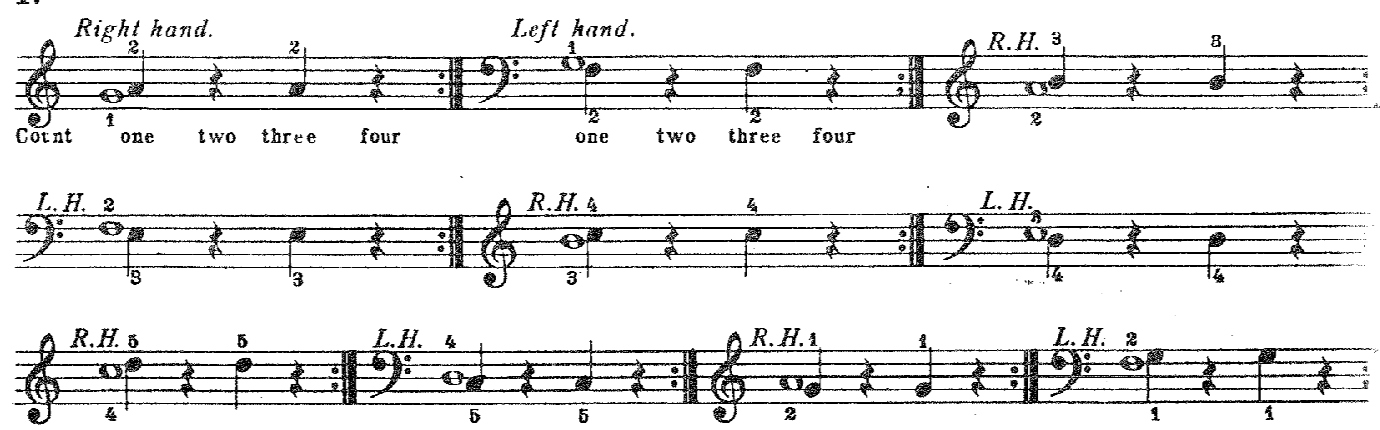

Figure 1. Oscar Beringer- A Complete Pianoforte Tutor pg. 6

In the first exercises played with the instructor after the first two exercises, the left hand immediately starts playing with the fifth finger. The third finger exercises on both hands are used only in exercises that teach note values, and there is no other exercises. Most of the musical pieces used in the book do not belong to the composer, instead the selected works of other composers are used.

\subsection{Bartok - Reschofsky (Zongora Iskola- Hungary, 1913)}

The correct hand and wrist positions are illustrated at the beginning of the book. Among the examined methods, the visuals of the position of the hand in the change of finger numbers are only available in this method.

Zongora Iskola starts with separate hand exercises. The left hand starts with the fifth finger and moves on to different finger numbers after the 25th exercise.

As seen in exercise 27, jumping from note to note is practiced only with the fifth finger of the left hand. The right hand does the same with the fifth finger. Although it is noted that the same exercise should be done with other finger numbers, starting this exercise with the fifth finger on both hands is something not encountered in other methods. Playing such exercises without strengthening the fifth finger on the left hand involves technical risks. The reason for this is that it is difficult to play without changing the natural position of the hand, especially when playing with only the fifth finger of the left hand.

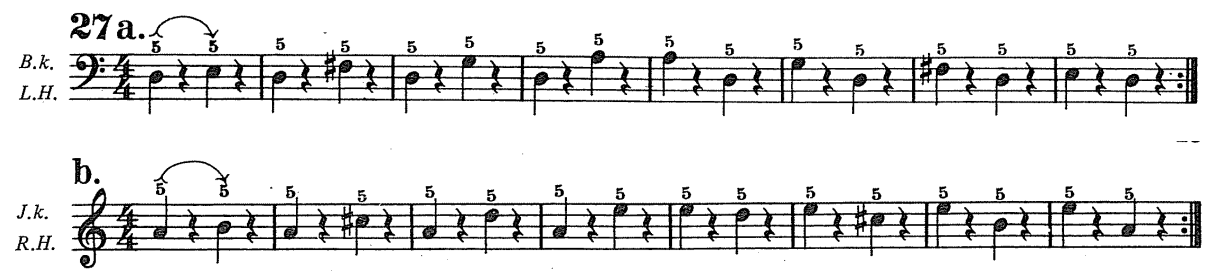

Figure 2. Bartok - Reschofsky- Zongora Iskola pgs. 22-23

In the 28th exercise, while the left hand is practicing jumping from the first finger to other finger numbers, the right hand is jumping from the fifth finger to other finger numbers. It is quite remarkable that in this exercise, both hands are played at the same time and that the ascending exercise is also performed in the opposite (descending) direction. Therefore, this exercise is not only a finger exercise, but also a brain exercise.

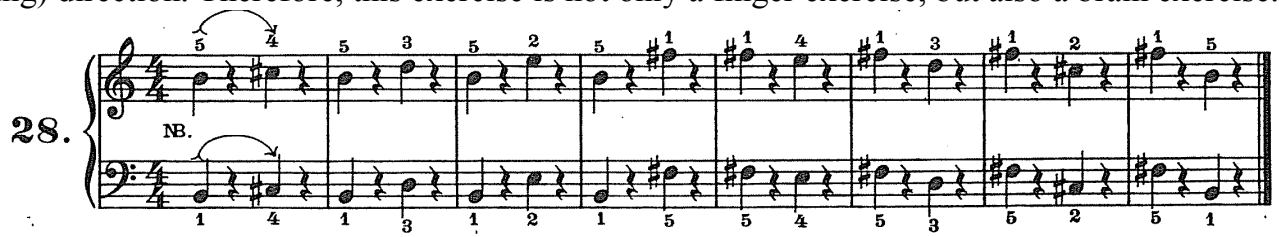

Figure 3. Bartok - Reschofsky- Zongora Iskola pg.23

Except for the last few exercises, the exercises used in the book are all original. Apart from the legato and staccato exercises in each method, all kinds of rhythmic and technical exercises were carried out in detail. Even the necessity of playing staccato at the end of slurs is exemplified.

3.3 Maria Cernovodeanu (Mica Metoda de Pian- Romania, 1981)

Of all the methods researched for this article, this is the only book describing the internal mechanism of the piano. Although there are many methods that show correct and incorrect hand positions, Maria Cernovodeanu's method also describes incorrect sitting positions. In general, popular melodies and children's songs are used in the book. 
The first exercise is the third finger 'catwalk' exercise mentioned in the 'Learning the Hand Position' section. It starts with two clefs of middle C.

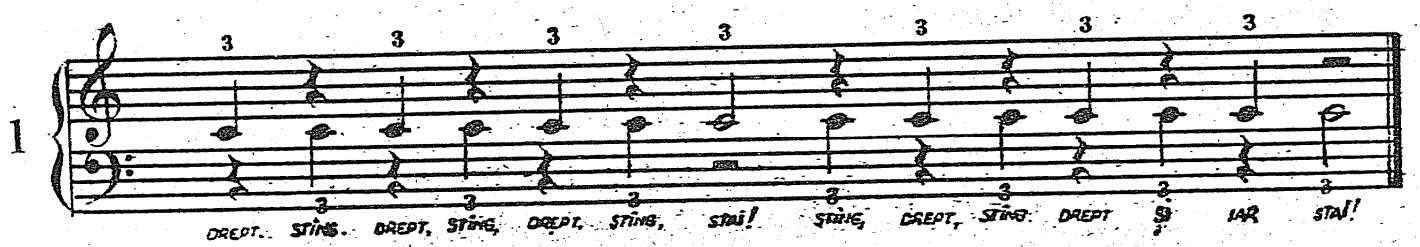

Figure 4. Maria Cernovodeanu- Mica Metoda de Pian exercise no.1

In the second exercise, different notes are played, but only the third finger is used. The third and fourth exercise starts with the second finger on both hands. Then the exercises continue with the third finger and mixed finger numbers. The fifth finger on the left hand is not used until the ninth exercise. Even after this exercise, the use of the fifth finger on the left hand is very limited, except for harmonic intervals. This shows that the principle of not using the weak fingers on the left hand without strengthening the fingers is adopted.

\subsection{J. Alex Burkard (Neue Anleitung für das Klavierspiel Band I- Germany 1961)}

This book is one of the most used methods in contemporary piano education. Both the right and wrong positions of the hand are given. In the method, both hands start playing from the middle $\mathrm{C}$ with the first finger. Both hands play together in the same exercise alternately. Immediately in the third exercise, the left hand descends to the note $\mathrm{B}$ with the second finger. In the sixth exercise, the third finger on both hands is added. Other finger numbers are quickly added to the others in the ensuing exercises.

In the first part of the method, both hands are designed in the natural position of the hand, with one note per finger without skipping. The right hand goes only from middle $\mathrm{C}$ to the $\mathrm{G}$. The left hand descends from middle $\mathrm{C}$ to the F. Despite this ease of posture, the content of the exercises progresses quickly and becomes complex quickly. Since Burkard provides such ease in posture, he does not think it is necessary to put finger numbers until the second part, when the exercises start to get difficult.

In the second part, the five-finger posture in general does not change much. However, from time to time, techniques such as octave jumps and transitioning from under the third finger to the first finger or over the first finger to the third finger are used too early. The difficulty level increases when accidentals and harmonic intervals are added to these from time to time. When it comes to the 36th exercise, it is very interesting to observe that the quarter notes used until this exercise suddenly switch to the eighth notes and chords, but immediately after this they revert back to simple notation.
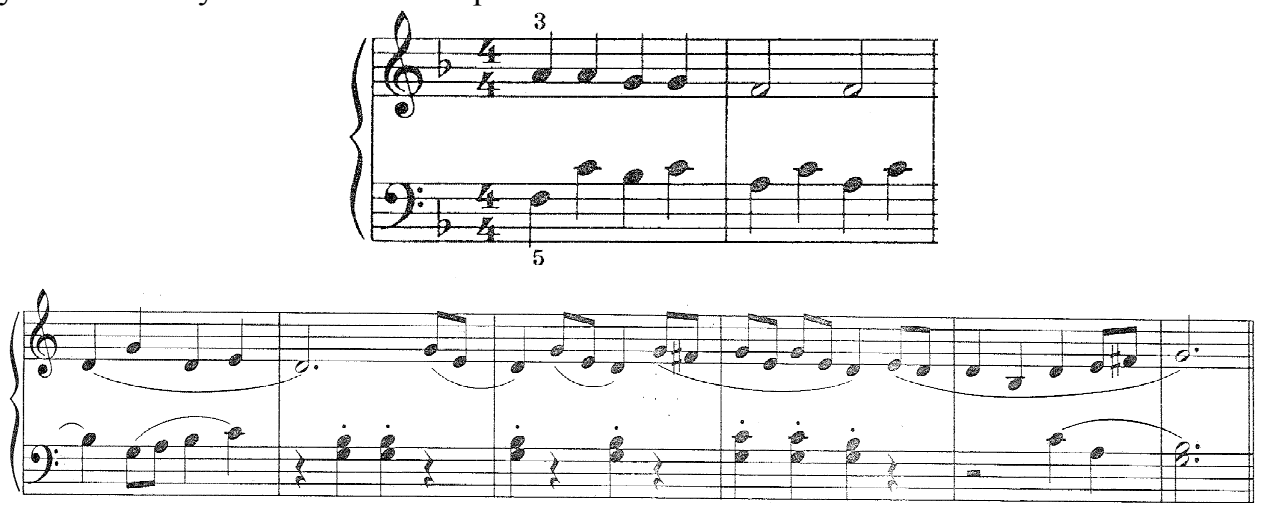

Figure 5. J. Alex Burkard-Neue Anleitung für das Klavierspiel Band I, Exercise no: 35 -36

From the left hand perspective, the fifth finger was used very little, especially at the beginning of the exercises, until the third part. This shows that although everything is progressing rapidly in the method, Burkard adopts the view that the fifth finger should not be used without strengthening the left hand.

\subsection{Sezgin Dinç (Step-by-Step Piano Method for Beginners, Turkey 2018)}

At the beginning of the book, correct hand holding images are presented along with detailed information. The first exercises start from the middle $\mathrm{C}$ in different clefs with separate hands. First, both hands use the first fingers. Other notes are added gradually in the following exercises, in a non-hurried instructional approach.

By the thirty-eighth page of the book, the right hand uses the fifth finger for the first time. The left uses the fifth finger on page forty-second for the first time.

Compared to the other methods in this book, the exercises of playing the two hands in turn are given much more attention and coverage. Rather than playing both hands at the same time, the focus is on basic knowledge 
(such as note values, legato). The two hands play together for the first time on page forty-eight.

In this method, all finger numbers are of equal importance. An equal quantity of exercise is presented each time a new finger is added. This introduces the use of the fifth finger on both hands quite late, delaying the use of the weakest fingers. In this way, it may have aimed to contribute to the strong position of the hand.

In addition to the known melodies, there are many original exercises in the book. All the pieces in the book are in $\mathrm{C}$ major. It is observed that the tones of the known works are also transposed to the $\mathrm{C}$ major, which is easy to play, for sake of convenience for the student, as in J. S. Bach's Musette in Musette D major.

\section{MUSETTE}

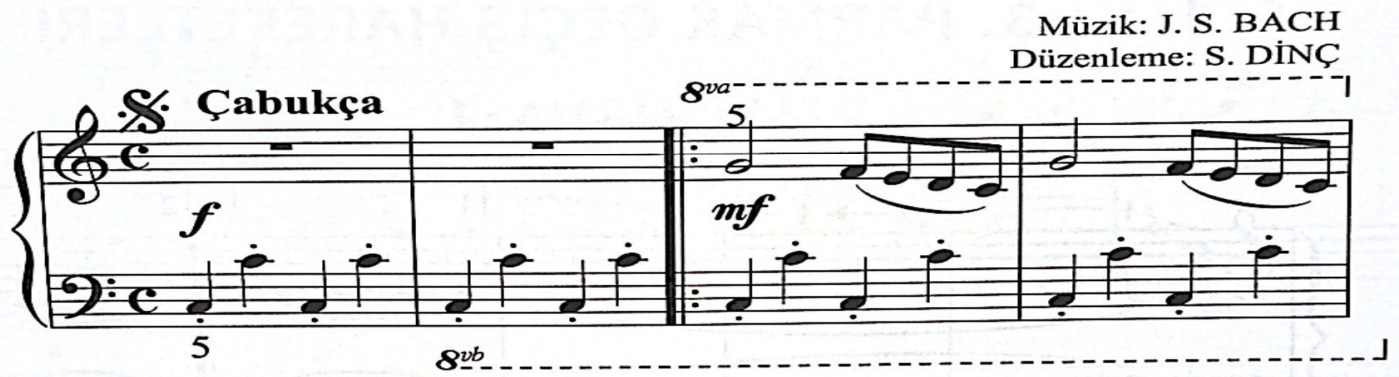

Figure 6. Sezgin Dinç- Step-by-Step Piano Method for Beginners, pg. 93

\section{Conclusion}

Finger numbers are of great importance especially in terms of the correct position of the left hand at the beginning of piano education. In terms of the initial exercises, use of first and fifth fingers was found in four of the five methods examined, and use of the third finger was identified in one of them. The fact that the use of the third finger, which is necessary for learning the right posture of the left hand, occurs in only one of the five methods, supports the idea of the necessity of doing multi-method work. In the left hand-oriented approach to piano training, the third-finger exercises should be performed first, followed by exercises involving the other fingers. At the same time, the teacher can use an exercise by adapting the finger numbers to the student when $\mathrm{s} / \mathrm{he}$ deems it necessary and when the piece is appropriate, regardless of which finger number is written. The teacher may be favoring the use of the third finger on the left hand, but this method may not be given much attention in the methods. In such a case, the teacher may choose to write an exercise or convert the exercise.

The exact way of implementing multiple methods is directly dependent on the educational approach favored by the instructor and the ability of the student. In the process of continuing the initial training through a single method, whether the instructor conducts the training quickly or slowly is related to the student's ability as well as the content of the method and the number of exercises on certain subjects. Instead, it would be appropriate to adopt a multi-method training approach, especially after the initial lessons for the left hand are taught by following a specific method or independently of any method.

\section{References}

Bartok - Reschofsky. (1964), "Zongora Iskola” Budapest: Editio Musica Budapest.

Beringer, O. (?), "A Complete Pianoforte Tutor" England: London: Bosworth \& Co. Ltd.

Burkard, J. A. (1961), “Neue Anleitung für das Klavierspiel”' Germany: B. Schott's Söhne Mainz.

Cernovodeanu, M. (1981), "Mica Metoda de Pian" Bucharest: Editura Muzicala.

Dinç, S. (2018), "Yeni Başlayanlar Iç̧in Adım Adım Piyano Metodu (Step-by-Step Piano Method for Beginners)" Ankara: Arkadaş Yayınevi. 